\title{
Percepção de enfermeiros acerca de lesões de pele em um hospital pediátrico no
}

\section{interior nordestino}

\author{
Nurses' perception about skin injuries in a pediatric hospital in the northeastern countryside \\ Percepción de enfermeros sobre lesiones cutáneas en un hospital pediátrico del nordeste del campo
}

Recebido: 20/01/2022 | Revisado: 25/01/2022 | Aceito: 04/02/2022 | Publicado: 06/02/2022

\author{
Állif Ramon Lima Felix Da Silva \\ ORCID: https://orcid.org/0000-0001-9526-7311 \\ Hospital Infantil Martagão Gesteira, Brasil \\ E-mail: allif15-felix2010@ hotmail.com \\ Cinthia Reis Almeida \\ ORCID: https://orcid.org/0000-0001-7151-4832 \\ Hospital Infantil Martagão Gesteira, Brasil \\ E-mail: cinthiialmeida@gmail.com \\ Ivana Souza Freitas \\ ORCID: https://orcid.org/0000-0003-1887-0066 \\ Hospital Infantil Martagão Gesteira, Brasil \\ E-mail: cirurgica.pa@martagaogesteira.org.br \\ Lívia Pereira Pinheiro \\ ORCID: https://orcid.org/0000-0003-3587-6523 \\ Universidade do Estado da Bahia, Brasil \\ E-mail: líviappenf@gmail.com
}

\begin{abstract}
Resumo
Este estudo objetivou identificar a percepção dos enfermeiros acerca de lesões de pele desenvolvidas em um Hospital pediátrico de Salvador Bahia. Método: Trata-se de pesquisa qualitativa, sendo que, na coleta de dados, recorreu-se à entrevista com roteiro previamente elaborado e à análise de discurso para sistematizar o material obtido. Resultados: os dados evidenciam que os enfermeiros têm domínio acerca das técnicas para prevenção de lesões e destacam a importância da educação continuada no cuidado a saúde. Conclusão: As peculiaridades dos profissionais entrevistados permitem identificar indivíduos graduados há mais de 2 anos, $90 \%$ do sexo feminino e $70 \%$ com idade entre 31 a 40 anos. Através das falas observou-se a importância do AGE em tais lesões, com o objetivo principal de nutrição e hidratação da ferida, corroborando com os achados na literatura., observa-se a inquietude dos profissionais na busca por uma comissão, um núcleo que pudesse dar suporte na tomada de decisões. Percebeu-se a necessidade de uma educação continuada principalmente pelas mudanças constantes na saúde, uma comissão de pele efetiva para dar suporte e orientação sobre essas lesões e observou-se através das falas que a ausência de materiais pode prejudicar na continuidade do tratamento.
\end{abstract}

Palavras-chave: Enfermagem; Assistência; Lesões de pele.

\begin{abstract}
This study aimed to identify nurses' perception of skin lesions developed in a pediatric hospital in Salvador Bahia. Method: This is a qualitative research, and, in the data collection, we used the interview with a previously prepared script and the discourse analysis to systematize the material obtained. Results: the data show that nurses have mastery of techniques for injury prevention and highlight the importance of continuing education in health care. Conclusion: The peculiarities of the professionals interviewed make it possible to identify individuals who graduated for more than 2 years, $90 \%$ female and $70 \%$ aged between 31 and 40 years. Through the speeches, the importance of EFA in such injuries was observed, with the main objective of nutrition and hydration of the wound, corroborating the findings in the literature. support in decision making. It was noticed the need for continuing education mainly due to the constant changes in health, an effective skin commission to support and guide these lesions and it was observed through the speeches that the absence of materials can harm the continuity of treatment.
\end{abstract}

Keywords: Nursing; Assistance; Skin lesions.

\section{Resumen}

Este estudio tuvo como objetivo identificar la percepción de las enfermeras sobre las lesiones cutáneas desarrolladas en un hospital pediátrico de Salvador Bahía. Método: Se trata de una investigación cualitativa, y en la recolección de datos se utilizó la entrevista con guión previamente elaborado y el análisis del discurso para sistematizar el material obtenido. Resultados: los datos muestran que los enfermeros tienen dominio de las técnicas para la prevención de 
lesiones y destacan la importancia de la educación continua en el cuidado de la salud. Conclusión: Las peculiaridades de los profesionales entrevistados permiten identificar individuos con más de 2 años de formación, el $90 \%$ del sexo femenino y el 70\% con edades entre 31 y 40 años. A través de las charlas se observó la importancia de los AGE en este tipo de lesiones, teniendo como principal objetivo la nutrición e hidratación de la herida, corroborando lo encontrado en la literatura apoyo en la toma de decisiones Se percibió la necesidad de educación continua principalmente por la constante cambios en la salud, una comisión de piel eficaz para apoyar y orientar estas lesiones y se observó a través de los discursos que la ausencia de materiales puede perjudicar la continuidad del tratamiento.

Palabras clave: Enfermería; Asistencia; Lesiones de la piel.

\section{Introdução}

As lesões de pele são consideradas um agravo a Saúde no Brasil desde os tempos remotos, sendo caracterizado como um problema de saúde pública no país. Isso pode ser evidenciado nos registros obtidos ao longo dos anos de milhões de pessoas que possuem algum tipo de lesão (Silva et al., 2017). No que tange a pediatria é comumente observado a presença de lesões e/ou feridas, visto a estrutura da pele, sensibilidade, funções ainda imaturas, principalmente quando comparados a pele de adultos. Apesar de tais evidências, há poucos estudos a respeito da ocorrência de lesões em crianças e recém-nascidos (RN) hospitalizados, desse modo a abordagem a população pediátrica exige conhecimento específico e tratamentos baseados em evidências (Santos \& Costa, 2015). No Brasil, problemas dermatológicos constituem cerca de 30\% do total de consultas na pediatria (Aires, 2015). Uma lacuna na literatura é evidenciada no que tange a pesquisas que analisem a prevalência, prevenção e tratamento de lesões de pele na pediatria, o que é imprescindível no campo da saúde. Em um estudo realizado em quatro hospitais da Suíça, identificou-se a prevalência de 27,7\% de lesões na pediatria (Schuler, et al, 2013). Na Jordânia estimou-se cerca de 6,6\% (Habiballah \& Tubaishat, 2016), dados esses que precisam ser expandidos entre países. No que concerne ao Brasil, no Hospital das Clínicas do Paraná encontrou a prevalência de 8,06\% (Crozeta, et al., 2010). No que se refere a incidência, uma pesquisa realizada no Ceará, em uma Unidade de Terapia Intensiva (UTI), encontrou 42,5\% (Carvalho, et al, 2011). Ressalta-se que é de suma importância a existência de estudos que forneçam dados de prevalência e evidência científica sobre as lesões na pediatria, principalmente para melhoria da assistência, baseada na prevenção, promoção e recuperação da saúde. Isto posto, a assistência á pacientes que possuem algum tipo de lesão é um desafio multiprofissional no campo da saúde, desse modo salienta-se a importância do cuidado do profissional enfermeiro em sua prática, sendo este o responsável pela realização dos curativos, que por sua vez deve ser realizado de modo holístico e integral, considerando o cliente um ser biopsicossocial, o que perpassa a mera técnica (Faria, et al., 2016). No que concerne a lesões, sua etiologia pode ser diversa, podemos citar: problemas de pele congênitas, utilização de dispositivos invasivos, extravasamento e/ou infiltração de soluções intravenosas, uso de substâncias químicas irritantes ou vesicantes, principalmente na área de oncologia, dermatites, imobilidade física, entre outras (Câmara, 2016). No Brasil, tem-se aumentado a comercialização de produtos e inovações no tratamento de feridas. Desse modo, é necessário conhecer tais produtos para utilizá-los da melhor maneira possível, baseado em evidência, salientando a atuação do enfermeiro na análise da melhor escolha, do custo benefício, da ação do tratamento e escolha terapêutica, na tomada de decisões objetivando a cicatrização da lesão. No entanto, para o profissional estar embasado em sua prática é preciso dispor de conhecimento sobre os produtos e sua eficácia (Faria et al., 2016; Ferreira, et al., 2012). É imprescindível entender e assim tratar cada indivíduo de modo singular, personalizando o atendimento, respeitando as características individuais de cada um bem como todos os demais aspectos envolvidos: biopsicossociais, etiologia e localização da lesão, fatores de riscos, idade, comorbidades, nutrição, presença de exsudato, entre outros (Prado et al., 2016). Mediante o exposto, evidenciando a incidência de feridas nos Hospitais pediátricos, surgiu o interesse e a motivação de pesquisar a percepção dos enfermeiros acerca de lesões de pele. O presente estudo tem sua relevância à medida que notamos a incidência de lesões nos Hospitais pediátricos e a necessidade dos enfermeiros que são os profissionais que realizam a atividade, principalmente por conhecerem os produtos mais utilizados nas coberturas e suas ações. Nota-se também que o empirismo, 
muitas vezes utilizado na profissão deve ser agregado a partir das evidências científicas. O estudo justifica-se pela escassez de pesquisas na área e através deste contribuições futuras e subsídios para investimentos no que tange a lesões de pele poderão ser evidenciados.

\section{Metodologia}

Trata-se de um estudo descritivo com abordagem qualitativa realizado em um Hospital Pediátrico no município de Salvador. A pesquisa descritiva tem como principal objetivo descrever e interpretar os fatos observados, características ou ações de uma determinada população. Pode-se destacar que a mesma é realizada sem que o pesquisador interfira, de modo que haja apenas a observação das relações, causas ou consequências dos fenômenos, analisando e os descrevendo (Prodanov; Freitas, 2013; Sampieri et al., 2013). A abordagem qualitativa é a forma em que se predomina a interpretação dos fenômenos através da observação dos sujeitos que estão interferindo na realidade. Nesse tipo de estudo os dados não podem ser considerados apenas pela lógica e/ou estatística, no entanto, necessita da participação dinâmica do pesquisador que deverá ir ao campo realizando entrevistas e observação direta para sua coleta e análise de dados, reconhecendo a complexidade do seu objeto de estudo (Minayo, 2013; Prodanov \& Freitas, 2013). A referida Instituição, considerada de grande porte possui estrutura para cerca de 220 leitos. Anualmente realiza mais de 500 mil atendimentos, atendendo cerca de mais de 80 mil pacientes. O hospital tornou-se referência no estado, principalmente no que tange suas especialidades oferecidas, destacando áreas de alta complexidade como área oncológica, cardíaca e neurológica (Martagão Gesteira, 2021). O período da pesquisa ocorreu de fevereiro de 2020 á dezembro de 2020. Participaram da pesquisa 11 enfermeiros de 2 unidades de assistência intermediária. A escolha destes sujeitos foi feita em virtude de estes profissionais vivenciarem, cada um imerso no seu espaço de atuação, a realidade da assistência bem como por serem os responsáveis pela realização dos curativos. Cada profissional será descrito pela letra E para manter seu anonimato, ex: E1, E2, E3. O estudo estabeleceu como critérios de inclusão: ser enfermeiro assistencialista, ter no mínimo 6 meses de contrato na Instituição, período esse de adaptação e conhecimentos dos serviços da Unidade. Não participaram da pesquisa: profissionais que estão afastados por licença ou férias, os que se recusaram a participar da pesquisa e os que não estiveram disponíveis em até 5 tentativas para responder a entrevista no momento da coleta. Da amostra, foram excluídos 4 profissionais por estarem a menos de 5 meses na Instituição, 1 por incompatibilidade de tempo durante a coleta e 1 por ser colaboradora do estudo. Para coleta de dados, foi realizada uma entrevista semi-estruturada utilizando roteiro previamente. Buscando melhor interpretação e veracidade na análise dos dados, foi feita a gravação de todas as entrevistas sendo cada uma delas obtidas em horário agendado antecipadamente, em seguida cada entrevista foi transcrita de modo fidedigno, respeitando o sigilo e a identidade de cada entrevistado. Para a análise e organização de dados adotou-se a categorização temática. Deste modo, os dados obtidos através de entrevistas foram transcritas na íntegra, lidas de modo exaustivo e atentamente, em seguida recortou-se e agrupou as falas (unidades de análise) convergentes formando as unidades de significados e as categorias temáticas. Realizado o recorte, os dados foram classificados em três categorias, buscando compreender o objetivo do estudo. Isto posto, após análise completa do material realizou-se a elaboração de uma síntese de interpretação dos dados através de uma redação, dialogando com os objetivos, as indagações e pressupostos do estudo em questão. O estudo foi realizado conforme as exigências da Resolução № 466/12 do Conselho Nacional da Saúde, que trata de pesquisas envolvendo os seres humanos e dispõe sobre diversos aspectos éticos como a autonomia, beneficência, justiça, equidade, não maleficência, respeitando o sujeito e defendendo sua vulnerabilidade, trazendo o máximo de benefícios e o mínimo de danos e riscos, de modo a garantir igual consideração dos interesses envolvidos, sem perder o sentido sócio humanitário, além de fornecer o sujeito o sigilo das informações colhidas (Brasil, 2012). O presente estudo foi aprovado pelo Comitê de Ética e Pesquisa da Maternidade Climério de Oliveira (MCO) - Universidade Federal da Bahia (UFBA) através do 
parecer de $\mathrm{N}^{\mathrm{o}}$ 3.818.065. Os participantes receberam informações e esclarecimentos sobre a pesquisa proposta, através do Termo de Consentimento Livre e Esclarecido (TCLE).

\section{Resultados e Discussão}

As peculiaridades dos profissionais entrevistados permitem identificar indivíduos graduados há mais de 2 anos. Como mostra a Tabela 1, a seguir:

Tabela 1: Caracterização do perfil dos profissionais entrevistados. Salvador (BA), 2021.

\begin{tabular}{lll}
\hline $\begin{array}{l}\text { Características } \\
\text { Sexo (n=11) }\end{array}$ & $\mathbf{N}$ & $\mathbf{( \% )}$ \\
Feminino & 10 & 90,91 \\
Masculino & 01 & 9,09 \\
Idade & & \\
20 -30 anos & 03 & 27,27 \\
$31-40$ anos & 08 & 72,73 \\
Tempo de formação & 06 & 54,55 \\
$1-5$ anos & 05 & 45,45 \\
6-10 anos & & \\
Tempo de atuação & 07 & 63,64 \\
$1-5$ anos & 04 & 36,36 \\
6 - 10 anos & & \\
Capacitação na área & 00 & 00 \\
Sim & 11 & 100 \\
Não & & \\
Pós Graduação & 06 & 54,55 \\
Sim & 05 & 45,45 \\
Não &
\end{tabular}

Fonte: Coleta de dados, Salvador - Ba (2021).

O que caracteriza o trabalho do enfermeiro é o desenvolvimento de atividades no processo de trabalho, ou seja, é a singularidade com foco na assistência á saúde e suas atribuições gerenciais, além dos subprocessos que vão do cuidar, assistir, administrar, pesquisar, ensinar dentre outras atribuições. Desse modo é imprescindível ter o conhecimento, competências e habilidades para que o mesmo exerça seu papel de modo efetivo e resoluto (Chaves \& Tanaka, 2012). Ao analisarmos o perfil dos entrevistados podemos observar a prevalência de indivíduos do sexo feminino $(90,91 \%)$ para $(9,09 \%)$ do sexo masculino, cenário no qual é considerado predominante na área da saúde, em especial da enfermagem. O COFEN, nos mostra através de uma pesquisa que cerca de $85,1 \%$ da equipe de enfermagem é feminina, e 14,4\% masculina, dados que corroboram com os achados da presente pesquisa (COFEN, 2017). Ao analisarmos a idade dos participantes podemos observar uma prevalência de enfermeiros com idade de 20 a 30 anos $(27,27 \%)$ e de 31 a 40 anos (72,73\%). No Brasil, no que tange a enfermagem estima-se que $40 \%$ do seu contingente é formado por profissionais de idade entre 36 - 50 anos, seguido por indivíduos de 26 a 35 anos correspondente a $38 \%$ e cerca de $2,1 \%$ com profissionais com mais de 61 anos, caracterizando a enfermagem como jovem, considerando que no geral 25, 3\% tem mais de 30 anos de idade (COFEN, 2017). Ao analisarmos o tempo de atuação dos profissionais podemos visualizar indivíduos entre 1 a 7 anos de atuação na área, de modo que $(63,64 \%)$ deles tem até 5 anos de atuação e (36,36\%) deles acima de 5 anos. 


\section{Categorias temáticas}

\section{Prevenção de lesões por pressão}

Observou-se que os profissionais de enfermagem demonstram ter conhecimento sobre a importância de cuidados assistenciais para prevenção de lesões de pele na pediatria. Nas falas a seguir destacam-se os cuidados necessários para prevenir lesões:

\section{[...] Mudança de decúbito, hidratação, é.. Proteção de proeminências ósseas, acho que só [...] (E3)}

[...] geralmente a gente pede pra reforçar hidratação da pele, alguns pacientes que já apresentam lesão (...) algum creme, que já pode ser fornecido pela instituição, tenta manter alinhado com a equipe e a família quanto os cuidados $[\ldots](E 7)$

[...] descompressão da pele, curativo diário, hidratação, uso de alguma cobertura especial ou um age, prevenção, na verdade para evitar que essas lesões apareçam como hidrocolóide, colocar um filme em proeminências ósseas [...] (E1)

[...] realizar mudança de decúbito, hidratação, porquê tem pacientes que ficam acamados no leito e pode acontecer do surgimento de lesões. Por isso a gente sempre orienta a realizar mudança de decúbito pra evitar pressão nas proeminências e colchão caixa de ovo [...] (E4)

A Organização Mundial da Saúde (OMS) criou uma proposta com o objetivo de instituir e viabilizar ações que ofereçam melhor qualidade nos serviços de saúde e aumente a segurança, sendo caracterizada como Aliança Mundial para segurança do paciente, o qual o Brasil integra (OMS, 2014). Mediante isto, temos também a Portaria de $\mathrm{n}^{\circ} 529$ e a Resolução RDC n $n^{\circ} 36$, que abrangem e descrevem ferramentas para segurança do paciente nos diversos serviços de saúde baseados na fomentação, criação e validação de protocolos, guias e manuais (Brasil, 2013). A criança possui características fisiológicas, anatômicas e estruturais singulares, que durante seu processo de adoecimento e hospitalização, conforme sua gravidade, podem levar a complicações que prologam seu tratamento ou dificultam na sua melhora, dentre as quais podemos destacar as lesões de pele (Rocha Neta, et al., 2018). Ressalta-se que muitas dessas lesões podem ser evitadas, por meio da identificação de risco dos pacientes e através de uma avaliação do profissional envolvido nesse processo. Após a identificação do risco é importante a inspeção diária da pele e a adoção de medidas preventivas que propiciem melhora da circulação sanguínea, alívio de pressão em proeminências ósseas, descompressão, avaliar estado nutricional e necessidade de intervenção, hidratação da pele e garantir reposicionamento com a mudança de decúbito (Rocha Neta et al., 2018). Conforme visto nas falas, os enfermeiros demonstram conhecimentos referentes a prevenção de lesões de pele na pediatria, de modo a trazerem dados de suas ações pertinentes que corroboram com os achados na literatura. Levando em consideração tais aspectos, ressalta-se a importância do exame físico realizado pelo enfermeiro e sua prescrição de cuidados, de modo holístico, criterioso, para prevenção dessas lesões. A inclusão do diagnóstico de enfermagem Risco para lesão por pressão, incluídos à terminologia North American Nursing Diagnosis Association (NANDA), 2018-2020, também denotam a sua importância para a enfermagem (Souza et al., 2018). O conhecimento da singularidade e dos aspectos que caracterizam a pediatria são de suma importância, conhecer a microbiota da pele, os tipos de alterações da integridade da pele, avaliação da lesão, criticidade na escolha da cobertura da lesão, conhecimento do mecanismo de ação das principais coberturas (papaína, hidrocolóide, hidrogel, alginato de cálcio, membranas ou filmes semipermeáveis, Ácidos Graxos Essenciais); executar as ações primordiais para cuidados com as lesões observadas, dentre outras, são conhecimentos imprescindíveis para o enfermeiro desde o início de sua formação na academia (Fontanele \& Cardoso 2011). 


\section{Utilização do age em lesões}

Ao serem questionados sobre a utilização do AGE as falas ressaltam sua importância e eficácia na pele íntegra como forma de prevenção de lesão e na pele rompida como favorecimento de hidratação e cicatrização como nas falas abaixo:

\section{[...] eu acho que o age é. Ele age regenerando os tecidos, hidratando [...] (E2)}

[...] é, a gente usa o termo hidratar, o local, a gente sabe que ele não é adequado pra um local que tem ferimento, aberto, não é indicado, mas ele tem um efeito muito bom no processo final da cicatrização [...] (E10).

[...] botando na balança é bastante positivo, eu vejo que ele não tem uma eficácia em pacientes que não tem lesão que poderia ser resolvido, com uma loção, uma hidratação e também a questão da criança, que ela consegue fazer essa reposição hídrica, pela alimentação ou venóclise quando não é um paciente desidratado [...] (E7).

[...] atua principalmente na hidratação e na produção de novas. Como chama? Esqueci... Na cicatrização do local $[\ldots](E 1)$.

[...] tem a finalidade de cicatrização, ele ajuda muito, ao meu ver... Principalmente lesões de grau leve que não tem esfacelo, tecido necrosado... é pele íntegra pra hidratar, e pra massagem pra os pacientes [...] (E3).

Os óleos de origem vegetal são utilizados em lesões, nota-se principalmente nos países da América Latina. Estes óleos possuem em sua composição ácidos graxos, sendo o oleico, linoleico e linolênico, os mais abundantes. As principais funções dos ácidos graxos são: atuar como mensageiros intracelulares, gerando a adenosina trifosfato (ATP) após oxidação, atuam também como componentes que estruturam as membranas biológicas (Ferreira et al., 2012). Através dos achados, 100\% dos entrevistados demonstraram que a utilização do AGE em lesões, auxilia no processo de regeneração e cicatrização, além de hidratação, conforme mostrado pelos dados científicos. A função dos AGEs no tratamento e prevenção de lesões se configura devido a capacidade de formar uma barreira protetora na pele, que impede a maceração, além de atuar nos processos de inflamação celular, agindo e nutrindo os tecidos além de ter da capacidade de proporcionar a regeneração dos tecidos. Sua atuação consiste também no aumento da resposta imune do organismo no local afetado, acelerando o processo inflamatório e consequentemente estimulando através da angiogênese e epitelização a cicatrização, proporcionando e facilitando a entrada dos fatores de crescimento celular (Ferreira et al., 2012). Os AGES, podem ser aplicados de diversas maneiras, na pele íntegra ou na pele lesionada. Quando utilizado de modo tópico, sobre a pele íntegra ele tem alto poder de absorção, melhora nutrição, forma uma barreira que previne escoriação, promovendo hidratação e aumentando elasticidade da pele. Nos tecidos eles agem na rápida regeneração dos tecidos (Silva \& Silva, 2013).

\section{Problemáticas levantadas pelos entrevistados}

A análise da terceira categoria trata-se das problemáticas relacionadas a assistência de lesões na pediatria. Os resultados estão apresentados em três subcategorias, sendo elas: a importância da educação permanente, a importância da existência de uma comissão de feridas e a necessidade de insumos disponíveis.

A educação permanente demonstra-se essencial no processo do cuidar

[...] sim, preciso, acho que todos nós, enquanto enfermeiros, precisamos nos preparar pra essa questão do cuidado e tratamento de feridas, por que é algo que a gente vê o tempo inteiro, seria interessante que até a própria unidade, instituição dispusesse de um curso pra gente, se fosse possível, mas acredito que pra mim, seria interessante fazer. Por que é algo que a gente leva pra nossa vida [...] (E8)

[...] sim, eu acho que poderia ter sim uma capacitação, uma interação melhor, uma comissão que seja direcionada a esse assunto, apesar que aqui a gente tem bons resultados (...), mas acredito que sim poderia ter rodas de conversas, debates, trocas de experiências relacionadas aos assuntos e até de novos medicamentos e novos curativos [...] (E10) 
[...] acho que deveria ter um comitê aqui, e ter educação continuada, está sempre atualizando, ter uma educação permanente, continuada, pra que melhore a assistência dos profissionais da saúde em lesões [...] (E9).

É notório que a saúde está em constante mudança e desenvolvimento, e a enfermagem enquanto ciência necessita de constante atualização e modernização, principalmente do que tange a educação continuada, que se caracteriza como o aprimoramento dos recursos humanos, dos saberes e práticas, fornecendo aos sujeitos conhecimento para aprimoramento de suas ações (Carvalho, 2020). A educação continuada propicia ao profissional de saúde o conhecimento mais amplo e abrangente de sua realidade local, entendendo que para que ocorra transformações é necessário entender o cenário que se encontra, elencar problematizações e ofertar estratégias de mudanças. Ressalta-se que a educação continuada é um espaço de troca de saberes, de atualização de conhecimento, reflexão de ações e avaliação do cuidar (Pereira et al., 2018). A educação continuada abrange toda a equipe de enfermagem e reflete as demandas de cada serviço, considerando suas peculiaridades. Seu objetivo primordial é nortear a assistência principalmente devido o aparecimento de atualizações, novidades tecnológicas de modo a direcionar as ações da equipe (Carvalho, 2020). Diante do exposto, o processo educativo não deve ser entendido como algo pontual, pelo contrário, deve ser visto como algo contínuo, que tem o poder transformador do ambiente de trabalho e que auxilia no processo de cuidar, como demonstrado, unanimemente, nas falas faz-se necessário investimento na educação continuada.

\section{A importância da existência de uma comissão de feridas}

Das falas emergidas observa-se a inquietude dos profissionais na busca por uma Comissão, um núcleo que pudesse dar suporte na tomada de decisões no que tange ao cuidado de lesões, que proporcionasse oficinas, cursos, discussões sobre a temática:

[...] eu acho que de fato aqui a gente poderia ter uma comissão de feridas, por que claro que nós da assistência, devido a nosso conhecimento vai sabendo algum tipo de cobertura, mas a gente não tem aquele conhecimento aprofundado, que aquela pessoa que venha fazer saber o que é ideal mesmo, então eu acho que uma comissão seria legal, pra fazer um acompanhamento[...] (E1)

[...] acho que orientação da equipe em relação ao tipo de material e ao tipo de ferida, por que aqui por exemplo, tem uma comissão de feridas, mas a gente da assistência, não tem tanto contato com o pessoal dessa equipe, de feridas, $e$ não tem uma orientação... Até tem um relatório pra utilizar, mas uma coisa é ver e ser orientada, outra coisa é ler um papel, por exemplo as meninas mais novas que estão chegando algumas, as vezes não tem conhecimento (...) (E6)

[...] eu acho que a princípio, o ideal seria uma comissão de feridas, com pessoas com especialização nisso, não só pela prática, por que a gente sabe que apesar do conhecimento prático a gente precisa do conhecimento teórico, precisa estar se atualizando nas novas coberturas de feridas [...] (E11)

Dos discursos acima evidencia-se a necessidade de um grupo específico para o tratamento das lesões, equipe capacitada para treinar e direcionar os enfermeiros quanto aos cuidados com as lesões, visto que se observa no perfil dos profissionais, idades e tempo de atuação diferentes e ausência de capacitação na temática. O objetivo de uma comissão consiste na minimização do surgimento de lesões e na assistência aos pacientes acometidos de modo a contemplar a avaliação e a necessidade do emprego de tratamento adequado e acompanhamento evolutivo dessas lesões, salientando-se a importância da equipe multiprofissional (Torres et al., 2018; Costa \& Machado, 2016). Das falas, alguns profissionais citam a existência de uma comissão no hospital, porém outros citam a necessidade em se ter uma comissão, logo, sugere-se o repasse de informações a respeito da comissão, como a mesma atua, quem faz parte e o modo que a mesma poderá contribuir na assistência. Mediante o exposto, é sabido da importância de além de uma comissão, da necessidade de capacitação é necessário também a disponibilidade de insumos para o tratamento adequado dessas lesões. 
A necessidade de insumos disponíveis para assistência

Das dificuldades citadas, observa-se que a falta de insumos pode impactar no processo de cuidar de lesões, como dito:

[...] do hospital, as vezes a falta de material, da demanda, da demora de compra e dos profissionais e capacitação[...] (E2)

[...] o que dificulta é o período de liberação também, por que muitas vezes não tem como liberar logo o alginato que a gente não tem no setor e tem que aguardar compra, mas só que isso infelizmente é normal de acontecer [...] (E4)

[...] esse hospital eu acho limitado no quesito de coberturas, a gente não tem variedade, principalmente quando se trata de proteção, então precisaria centralizar o tratamento dessas lesões [...] (E11)

A ausência de materiais, insumos, algumas coberturas, foram apontadas como uma das dificuldades na realização da assistência ao paciente com feridas, o que de fato, é um obstáculo a ser ultrapassado para que se cumpra a função e suas atribuições. Existem materiais específicos para cada tipo de lesão. Depois de avaliar a lesão, é preciso definir qual o tipo de material a ser utilizado. Ressalta-se também a necessidade dos profissionais conhecerem a sua realidade e os materiais disponíveis para utilizarem de modo crítico, conforme demanda do paciente.

\section{Considerações Finais}

O profissional enfermeiro, juntamente com sua equipe é de fundamental importância no processo de tratamento e assistência a pacientes com lesões de pele, visto que é o profissional responsável pela realização dos curativos em um processo gradual e ativo, portanto, é imprescindível que o mesmo tenha domínio teórico e prático para um acompanhamento eficaz (Silva, et al., 2017). O presente estudo, ao analisar a percepção do profissional enfermeiro na assistência a pacientes com lesões, evidencia o domínio sobre as técnicas para prevenção das lesões no âmbito hospitalar. Através das falas observou-se a importância do AGE em tais lesões, com o objetivo principal de nutrição e hidratação da ferida, corroborando com os achados na literatura. Percebeu-se a necessidade de uma educação continuada principalmente pelas mudanças constantes na saúde, uma comissão de pele efetiva para dar suporte e orientação sobre essas lesões e observou-se através das falas que a ausência de materiais pode prejudicar na continuidade do tratamento. Sugere-se que as Instituições de saúde estabeleçam maneiras de discutirem as lesões e o tratamento delas, através de estudos de caso, rodas de conversas, discussão de artigos, para que haja troca de saberes. É importante que se elabore uma comissão de feridas e que ela dê o suporte necessário e que possa atualizar os profissionais sobre coberturas, suas ações e a necessidade de utilizá-la. Fomenta-se a necessidade de novos estudos nos mais diversos campos e cenários do que tange a pediatria e neonatologia, como exemplo das unidades existentes nos hospitais seja unidade de terapia intensiva, neonatal ou em unidades abertas de assistência visto suas especificidades, bem como as realidades observadas nas mais diversas Instituições no Brasil e mundo e a necessidade de compartilhar tais vivências e cenários. Conclui-se que além do conhecimento técnico e científico, é necessário que os enfermeiros sejam capacitados e aperfeiçoados no processo de assistência ao paciente com algum tipo de lesão, conhecendo a sua realidade e os materiais disponíveis, bem como sua atuação, ressaltando a importância de uma comissão bem como de um núcleo de educação continuada.

\section{Referências}

Azevedo, I. C., Costa, R. K. S \& Ferreira Júnior, M. A. (2018). Perfil da produção cientifica da enfermagem nacional sobre feridas. Revista cubana de enfermería. 34 (1). Revenfermeria.sld.cu/index.php/enf/article/view/1440/339.

Aires, S. T. (2015). Diagnóstico diferencial entre dermatoses e alergia: abordagem prática. Sociedade brasileira de pediatria- SBP. Departamento científico de alergia e imunologia. http://www.sbp.com.br/src/uploads/2015/02/texto_diag_dif_dermatoses-suzana2012.pdf. 
Brasil. (2012). Ministério da saúde. Conselho Nacional de Saúde. Comissão Nacional de Ética e Pesquisa - CONEP. Resolução 466/12. Sobre pesquisa com seres humanos. Brasília - DF http://bvsms.saude.gov.br/bvs/saudelegis/cns/2013/res0466_12_12_2012.html.

Brasil. (2013). Portaria $N^{\circ} 529$ de $1^{\circ}$ de Abril de 2013. Institui o Programa Nacional de Segurança do Paciente (PNPS). Ministério Da Saúde. Https://bvsms.saude.gov.br/bvs/saudelegis/gm/2013/prt0529_01_04_2013.html.

Câmara, S. M. C. (2016). Construção e validação de um protocolo de prevenção de pele para o recém-nascido em unidade de terapia intensiva neonatal. Dissertação de mestrado profissional em saúde da criança e do adolescente - Universidade Estadual do Ceará. Centro de ciências da saúde, Fortaleza.

Carvalho, G. B., Silva, F. A. A., Castro, M. E \& Florêncio, R. S. (2011). Epidemiologia e riscos associados à úlceras por pressão em crianças. Cogitare enfermagem. (16) 4, 640-6.

Carvalho, J. D. A. (2020). Importância da educação continuada em enfermagem. Rev. Saberes. (12) 1. https://facsaopaulo.edu.br/wpcontent/uploads/sites/16/2020/06/a-importancia-da-educa\%c3\%87\%c3\%83o-continuada-na-enfermagem..pdf.

Chaves, 1. D. P \& Tanaka, O. Y. (2012). O enfermeiro e a avaliação na gestão de sistemas de saúde. Rev. Esc. Enferm. USP (46) 5. https://www.scielo.br/scielo.php?script=sci_arttext\&pid=s0080-62342012000500033.

COFEN. (2017). Relatório final da pesquisa perfil da enfermagem no Brasil $\quad$ - Fiocruz/Cofen. (1) 2. http://www.cofen.gov.br/perfilenfermagem/pdfs/relatoriofinal.pdf

Crozeta, K., Stocco, J. G. D., Danski, M. T. R \& Meier, M. J. (2010). Úlceras por pressão em neonatos e crianças: perfil epidemiológico e clínico. Revista mineira de enfermagem. (14) 2, p 233-238.

Faria, G. B. G, et al. (2016). Conhecimento e prática dos enfermeiros sobre o cuidado com feridas. Revista enfermagem ufpe online. Recife, 10 (12).

Ferreira, A. M., Souza, B. M. V., Rigotti, M. A \& Loureiro, M. R. D. (2012) Utilização dos ácidos graxos no tratamento de feridas: uma revisão integrativa da literatura nacional. Rev. Esc. Enferm - usp. (46) 3. São Paulo. https://www.scielo.br/scielo.php?script=sci_arttext\&pid=s0080-62342012000300030.

Fontanele, F. C \& Cardoso, M. V. L. (2011). Lesões de pele em recém-nascidos no ambiente hospitalar: tipo, tamanho e área afetada. Rev. Esc. Enferm. Usp (45) 1. São Paulo. https://www.scielo.br/scielo.php?script=sci_arttext\&pid=s0080-62342011000100018.

Habiballah, L \& Tubaishat, A. (206). The prevalence of pressure ulcers in the paediatric population. Journal tissue viability. (25) 2.

Hospital Martagão Gesteira. (2016). Nossa história. Martagão gesteira. http://martagaogesteira.com.br/o-martagao/.

Minayo, Maria Cecília de Souza. (2010). Pesquisa social: teoria, método e criatividade. (29a ed.). https://editorialgaudencio.com.br/2013/01/02/maria-ceciliade-souza-minayo/.

Minayo, Maria Cecília de Souza. (2013). A produção de conhecimentos na interface entre as ciências sociais e humanas e a saúde coletiva. Saúde e sociedade. https://www.scielosp.org/article/sausoc/2013.v22n1/21-31/pt/.

Prado, A. R. A, et al. (2016). O saber do enfermeiro na indicação de coberturas no cuidado ao cliente com feridas. Estima. (14) 4. 175-182.

Pereira, L. Á., Silva, K. L., Andrade, M. F. L. B \& Cardoso, A. L. F. (2018). Educação permanente em saúde: uma prática possível. Rev enferm ufpe on line. Recife, 12(5). P. 1469-79. https://periodicos.ufpe.br/revistas/revistaenfermagem/article/viewfile/231116/29010.

Prodanov, C. C \& Freitas, E. C. (2013). Metodologia do trabalho científico: métodos e técnicas da pesquisa e do trabalho acadêmico. feevale.

Rocha Neta, A. P., Cavalcante, T. B., Lima, A. B. S., Maciel, S. M., Miranda, S. M \& Sousa, A. R. (2018). Caracterização epidemiológica e clínica de crianças com lesões por pressão. Revista enfermagem atual. (86).

Sampieri, R. H., Collado, C. F \& Lucio, M. P. B. (2013). Metodologia de pesquisa. (5a ed.), Penso.

Santos, V. S \& Costa, V. (2015). Prevenção de lesões de pele em recém-nascidos: o conhecimento da equipe de enfermagem. Texto contexto enferm, Florianópolis. 24 (3). https://www.scielo.br/pdf/tce/v24n3/pt_0104-0707-tce-24-03-00731.pdf.

Santos, K. K. (2016). Principais infecções cutâneas na infância: uma questão de educação em saúde na unidade básica de saúde sagrada família. Trabalho de conclusão de curso apresentado ao curso de especialização em estratégia saúde da família, universidade federal de minas gerais, para obtenção do certificado de especialista.

Schlüer, A. B., Schols, J. M \& Halfens, R. J. (2013). Pressure ulcer treatment in pediatric patients. Adv skin wound care, (26) 11. P. 504-10. Silva, A. C. O, et al. As principais coberturas utilizadas pelo enfermeiro. (2017). Revista uningá. (53) 2. P.117-123.

Silva, E. R \& Silva, F. E. (2013). Efeitos tópicos dos ácidos graxos essenciais (AGE) - ômega 3 e 6 aplicados em feridas abertas: uma revisão Bibliográfica. Faculdades integradas promove de Brasília. Curso de bacharel em enfermagem. Brasília. http://nippromove.hospedagemdesites.ws/anais_simposio/arquivos_up/documentos/artigos/46b8b96ffbb25f50ee1137945ec1a931.pdf

Souza, A. S., Mendonça, P. K., Loureiro, M. D. R \& Frota, O. P. (2018). Prevenção de lesão por pressão: ações prescritas por enfermeiros de centros de terapia intensiva. Contexto - enferm. (27) 4. Florianópolis. https://www.scielo.br/scielo.php?script=sci_arttext\&pid=s0104-07072018000400310.

Torres, R. C., Oliveira, S. J., Abud, A. C. F \& Rego, R. M. V. (2018). Implantação da comissão de prevenção e tratamento de lesões na pele em um hospital público. Revista enfermagem atual. (86) . http://reme.org.br/artigo/detalhes/1225http://revistaenfermagematual.com/arquivos/ed_86_revista_24/17.pdf.

World health organization (WHO). (2014). The conceptual framework for the international classification for patient safety. http://www.who.int/features/factfiles/patient_safety/en/ 